\title{
NEW ALGORITHMS TO CALIBRATE THE STRÖMGREN PHOTOMETRIC SYSTEM IN METAL-TO-HYDROGEN RATIO
}

\author{
V.MALYUTO \\ Tartu Astrophysical Observatory \\ EE2444, Tõravere, Estonia
}

The previous calibration of the Strömgren photometric indices in terms of spectroscopic $[\mathrm{Fe} / \mathrm{H}]$ values was performed by Schuster and Nissen (1989) with the application to their sample of calibration stars a mathematical package handling the regression of $[\mathrm{Fe} / \mathrm{H}]$ values against the $(b-y), m_{1}$, and $c_{1}$ indices, and their combinations. To provide a similar regression we applied a quite another independent statistical method (notably that of of stepwise linear regression) to the same sample and also to other available calibration samples of $\mathrm{F}$ stars. We conclude that in our case the quality of calibration was slightly better and the analytical form of calibration equation was much simpler.

To extend the sample of calibration stars, we supplemented the recent spectroscopic $[\mathrm{Fe} / \mathrm{H}]$ catalogue (compiled by Cayrel de Strobel et al. 1992) by newer data, together with some data overlooked by Cayrel de Strobel et al. Those $\mathrm{F}$ stars with $[\mathrm{Fe} / \mathrm{H}]>-1.0$ which were common to different sources were analysed with respect to their systematic and accidental differences in the $[\mathrm{Fe} / \mathrm{H}]$ values. An algorithm elaborated earlier to estimate the accuracy of stellar physical parameters from intercomparison of homogeneous samples (Malyuto, 1993) was applied.

\section{References}

Cayrel de Strobel, G., Hauck, B., Francois, P., Thevenin, F., Friel, E., Mermilliod, M., Borde, S. (1992) Catalogue of $[\mathrm{Fe} / \mathrm{H}]$ determinations: 1991 edition, Astronomy and Astrophysics Supplement Series, Vol. no. 95, pp. 273-336

Malyuto, V. (1993) Estimates of the accuracy of stellar physical parameteres from intercomparison of catalogues, Astronomy and Astrophysics, Vol. no. 278, pp. 73-80

Schuster, W., Nissen, P.E (1989) uvby $\beta$ photometry of high-velocity and metal-poor stars, Astronomy and Astrophysics, Vol. no. 221, pp. 65-77 